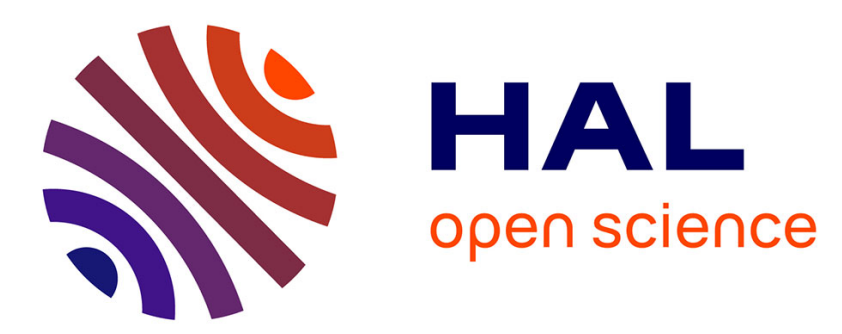

\title{
Emotional modulation of peripersonal space as a way to represent reachable and comfort areas
}

\author{
Marwen Belkaid, Nicolas Cuperlier, Philippe Gaussier
}

\section{To cite this version:}

Marwen Belkaid, Nicolas Cuperlier, Philippe Gaussier. Emotional modulation of peripersonal space as a way to represent reachable and comfort areas. IEEE International Conference on Intelligent Robots and Systems, Sep 2015, Hamburg, Germany. hal-01212393

\section{HAL Id: hal-01212393 \\ https://hal.science/hal-01212393}

Submitted on 6 Oct 2015

HAL is a multi-disciplinary open access archive for the deposit and dissemination of scientific research documents, whether they are published or not. The documents may come from teaching and research institutions in France or abroad, or from public or private research centers.
L'archive ouverte pluridisciplinaire HAL, est destinée au dépôt et à la diffusion de documents scientifiques de niveau recherche, publiés ou non, émanant des établissements d'enseignement et de recherche français ou étrangers, des laboratoires publics ou privés. 


\title{
Emotional modulation of peripersonal space as a way to represent reachable and comfort areas
}

\author{
Marwen Belkaid Nicolas Cuperlier Philippe Gaussier \\ Neurocybernetics team, ETIS laboratory, UMR 8051, CNRS/ENSEA/UCP \\ 95000 Cergy Cedex, France. \\ \{firstname.lastname\}@ensea.fr
}

\begin{abstract}
This work is based on the idea that, like in biological organisms, basic motivated behavior can be represented in terms of approach and avoidance. We propose a model for emotional modulation of the robot peripersonal space. That is to say, an area both reachable and secure; the space where the robot can act. The contribution of this paper is a generic model that integrates various stimuli to build a representation of reachable and comfort areas used to control robot behavior. Such an architecture is tested is three experiments using real robot and simulations. It is compared with two altered architecture versions. We show how our model allow the robot to solve various tasks, display emotionally colored behaviors and account for results from psychological studies.
\end{abstract}

\section{INTRODUCTION}

Reactive architectures offer an alternative to the deliberative paradigm [1][2]. Rather than top-down centralized decisions following a sense-plan-act sequence, they rely on sensorimotor behaviors. No need for an internal model of the world nor planning. The sense-act coupling allows for a fast control by only figuring out what to do in the next step. Yet, as soon as several behaviors are available, giving up the idea of building a plan to solve the task raises the problem of action selection.

Research in reactive systems largely took inspiration from ethology. For instance, the subsumption architecture organizes the sub-behavior into a hierarchy of layers, all running in parallel [2]. Although the higher levels may inhibit lower sub-behavior when needed, the idea is that they utilize lower competences in a bottom-up way. Moreover, loose hierarchy structure was proposed as an alternative to rigid winner-takes-all approaches in top-down hierarchical models [3]. Nevertheless, such architectures suppose a hard-coded priority between the sub-behaviors and offer little flexibility in terms of action selection adaptability.

It has been shown that it is possible to use parallel architectures without any preprogrammed top-down control over sub-behaviors [4]. Indeed, the system can learn what behavior is appropriate given a certain sensory input. Another example is to use reinforcement learning to implement a gating mechanism that arbitrates between different strategies based on previous rewards [5].

Dynamic Neural Fields (DNFs) present a set of properties (bifurcation, fusion, hysteresis, etc.) that make them a good candidate for robotic control [6]. Behaviors suggested by different strategies can be considered as weighted inputs of a
DNF, which output is used to determine the action to perform [6][7]. The inputs weights can be hardcoded (in order to define certain priorities) and/or modulated according to the situation. In [7], an emotional metacontroller weights subbehaviors importance in the action selection process based on prediction errors. Indeed, emotions being inherently part of control and self-regulation is becoming more and more admitted [8]. They express motivational states and foster conflict resolution [9][8]. Besides, the idea of modeling emotions and motivations in robotic architectures has been considerably studied [10][11]. We believe such an approach allows for gaining flexibility and solving various tasks.

In this paper, we propose a model in which emotions modulate the robot perception in order to change its behavior. We consider the case of perceiving the surrounding space. That is to say the comfort area where intrusions should be avoided and the reachable area where objects can be approached. Several evidence of the effect of emotions on such perceptions can be found in psychological and neuroscientific studies [12][13][14][15]. In our case, the robot emotional states depend on the dynamics of basic components: pleasure, pain and motivation [16]. Thus, it encompasses the affective and the motivational states, respectively determined by received rewards or punishments and by appetitive and defensive drives. The model proposes that these states modulate the perception of sensory input before and after they are integrated over time (like in a working memory). Thereby, we obtain a subjective and motivated representation of the surrounding space which is used to drive robots behavior.

In the next sections, we give some evidence of PPS plasticity from the literature and present our model for its emotional modulation. Next, we give some implementations details and expose the experimental paradigm we use to evaluate our model. Finally, we describe three experiments using real robots and simulations.

\section{PERIPERSONAL SPACE PLASTICITY IN APPROACH AND AVOIDANCE}

In the literature, the notions of comfort zone or personal space refer to the space needed to feel comfortable and safe [12][13]. On the other hand, the term reachable space is used in action-related contexts, e.g. moving or manipulating objects [14][15]. Although they can sometimes be 

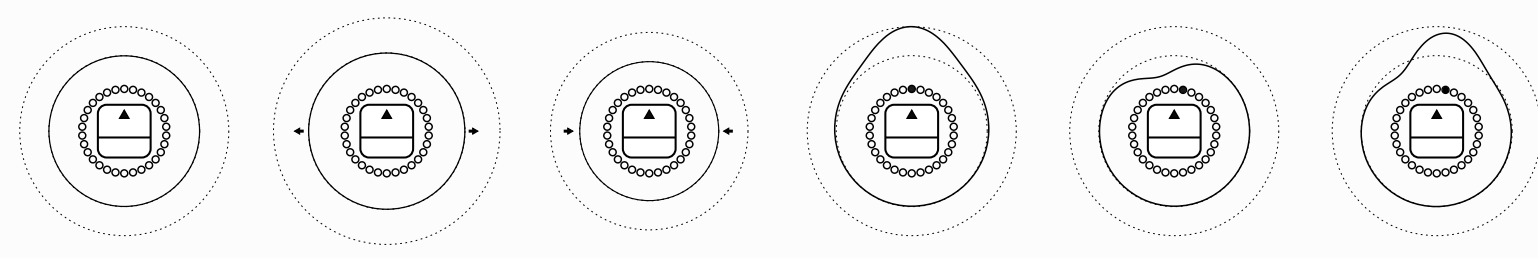

Fig. 1. Different forms of modulation of robot PPS. FAR-LEFT: No modulation. LEFT and CENTER-LEFT: The comfort zone contracts or dilates according to the pleasantness of the affective state. CENTER-RIGHT, RIGHT, and FAR-RIGHT: Also, appetitive and aversive stimuli respectively induce an extension or a retraction of the reachable space in the corresponding direction.

distinguished, we see these two notions as two aspects of the same process of integrating information to represent the space around oneself. It is the peripersonal space (PPS), as a multimodal sensorimotor interface between the body and the environment. It represents an area both reachable and secure. Thus, it can be used to avoid threatening intrusions or the approach desirable objects.

It has been demonstrated that PPS is plastic. Indeed, positively valenced objects tend to be perceived as more reachable than negative ones [14]. However, the presence of threatening objects in our peripersonal area can be perceived differently. For instance, a knife seems farther when oriented toward us, i.e. when potentially dangerous [15]. On the other hand, a positive affective state, induced by pleasant music for instance, can impact the PPS as well, reducing the area needed to feel comfortable in over-crowded spaces [13].

As a sensorimotor interface with the world which is used in both approach and avoidance behaviors, we suggest it is interesting to model PPS in a robotic system. Here, we are more precisely interested in its modulation by emotional states. If we consider a mobile robot in a navigation task, we can represent various states of its PPS perception like in Fig. 1. Indeed, its comfort zone can contract or dilate according to the pleasantness of the current affective state. Also, appetitive and aversive stimuli respectively induce an extension or a retraction of the reachable space in the corresponding direction.

\section{PROPOSED MODEL FOR EMOTIONAL MODULATION OF PERIPERSONAL SPACE}

We propose that PPS perception is based on a working memory (WM) that integrates sensorimotor information. For instance, the robot can remember the position of an obstacle it avoided. Also, it can update a path integration vector associated with a goal according to the speed and direction of instantaneous movement. We propose this sensorimotor input has to be integrated according to the current affective state. If the robot perceives a collision as a punishment signal, obstacles become more salient and leave a bigger trace in the (WM). Indeed, punishment-induced negative state expands the robot comfort zone [13].

Previous work presented an architecture allowing for handling a limited WM in tasks including multiple goals [17] (See Fig. 2 for an overview). No further understanding of the WM model is required for this paper given the small number of goals and sensory inputs in our experiments. We rather

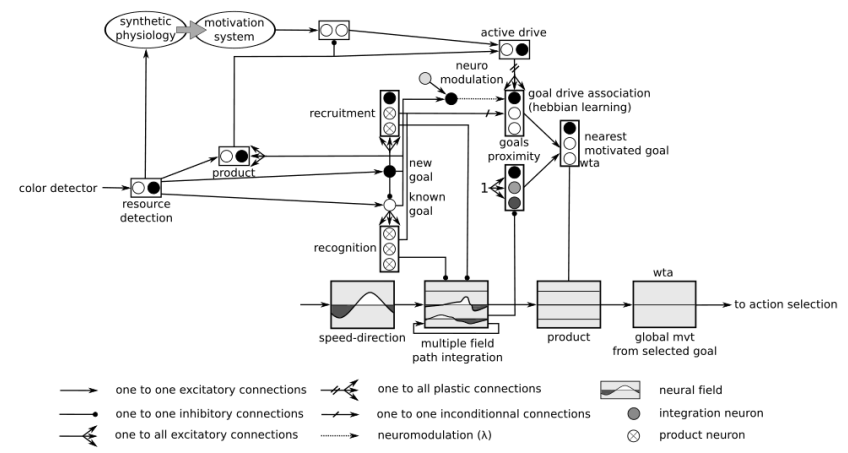

Fig. 2. Model of working memory (WM, from [17]). Upper part: Hebbian learning allows for associations between resources and the drives they satisfy. Lower part: Proprioceptive path integration fields in the WM encode the distance and direction of a set of goals, i.e. visited resource spots that satisfied a drive. Middle part: Resource detection resets the recornized WM field (known goal) or triggers field recruitement/replacement (new goal).

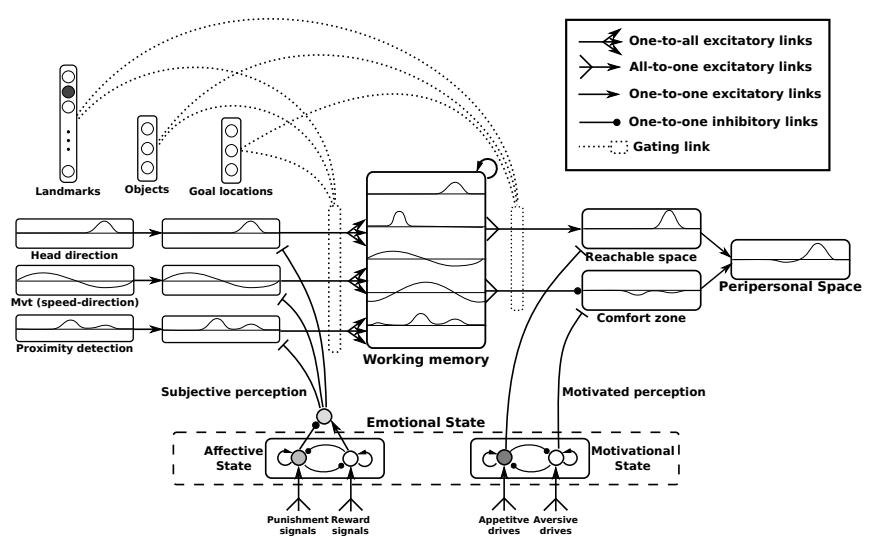

Fig. 3. Model for building a representation of the robot peripersonal space. It is based on working memory taking input from various sensory modalities. PPS is modulated by the robot emotional states in order to integrate a subjective and motivated perception of its environment.

focus on the emotional modulation at different levels of the information processing loop (See Fig. 3).

In this work, the robot emotional states depend on the dynamics of basic components: pleasure, pain and motivation [16]. It encompasses the affective and the motivational states. The former is determined by received rewards or punishments and the latter by appetitive and defensive drives. The model proposes that the affective state modulates the perception of sensory inputs before they are integrated in the WM. Then, relevant information from the WM is mod- 
ulated by the motivational state and merged to represent the robot peripersonal space. Such a subjective and motivated perception is used to determine robot actions, consequently influencing future sensations.

\section{STUDYING THE IMPACT OF EMOTIONAL MODULATION OF PERIPERSONAL SPACE}

\section{A. Method}

In this paper, we are interested in the emotional modulation of the peripersonal space. More specifically, we address the cases where approach and avoidance behavior are in contradiction. Thereby, we can observe the impact of the emotional state on the action selection process.

We use a dynamic neural field (DNF) [6] to merge information from WM in PPS representation. Appetitive and aversive stimuli given as input generate attractors and repulsors in the DNF. The potential $u$ of each neuron $x$ is updated as following:

$$
\begin{aligned}
\tau \cdot \frac{u(x, t)}{d t}= & -u(x, t)+i(x, t)+h \\
& +\int_{z \in V_{z}} w(z) \cdot f(u(x-z, t)) \cdot d z
\end{aligned}
$$

where $f(x)=\tanh (x)$ and is used to calculate the neuron actiity, $\tau$ is the time constant, $i$ the input, $h$ a constant inhibition potential and $w$ an interaction kernel. Using a DoG (Difference of Gaussian) function as the interaction kernel allows proximal stimuli to reinforce each other and to inhibit distant ones. The highest neuron activity is used to calculate the linear speed. Also, we calculate the rotational speed via a readout of the output derived signal (wrt current orientation).

In experiments 1 and 2 (Sect. V and VI), we study the single-resource case. The robot has one appetitive and one aversive drive - respectively feeding and protecting its own physical integrity. The feeding drive is determined by the level of a simulated physiological variable, based on the model of hypothalamus proposed in [18]. The level $r$ of the physiological variable associated to the resource at time $t$ is:

$$
r(t)=\alpha_{r}\left(r_{\max }-r(t-d t)\right) . I(t)-\beta_{r} r(t-d t)(1-I(t))
$$

where $r_{\max }$ is the maximal variable level (set to 1), $\alpha_{r}$ and $\beta_{r}$ respectively indicate the ingestion and the consumption speed factors and $I$ is the ingestion signal (1 when on the resource and $r(t)<r_{\max }$ and 0 otherwise). On the other hand, the safety drive mimics the role of the superior colliculus in the integration of multiple sensory input in order to trigger defensive behavior like avoidance or withdrawal [19]. In our case, we calculate the mean activity on the proximity sensors obtain the level $t h$ of threat at time $t$.

The perception of the threath is modulated according to the robot affective state. The latter depends on the received punishment and reward signals used to simulate pain and pleasure. In these experiments, they are respectively given by collision and resource detection. In order to obtain a midium-term state that integrates the two kinds of signals, we define the affective state $a$ according to punishments $a_{p n}$ and rewards $a_{r w}$ at time $t$ as following:

$$
\begin{aligned}
a(t) & =a_{r w}(t)-a_{p n}(t) \quad \text { with } \\
a_{p n}(t) & =\varepsilon_{a} \cdot a_{p n}(t-d t)-\gamma_{a} \cdot a_{r w}(t-d t) \\
a_{r w}(t) & =\varepsilon_{a} \cdot a_{r w}(t-d t)-\gamma_{a} \cdot a_{p n}(t-d t)
\end{aligned}
$$

where $\varepsilon_{a}$ and $\gamma_{a}$ respectively represent the integration and inhibition factors of the competition.

Starting from the resource location, the return vector is calculated by integrating the speed and direction of intantaneous movements. The activity $p$ of each neuron $x$ in the path integration field at time $t$ is given by the following equation:

$$
p_{x}(t)=\sum_{t_{r}}^{t}\left(s(t) \cdot \cos \left(\frac{d(t)+2 \pi x / n}{n}\right)\right) \cdot(1-R(t))
$$

where $t_{r}$ is the last reset time, $s$ the linear speed, $d$ the direction, $R$ the reset signal and $n$ the size of the neural field. Reset signals occur when the resource is detected.

The feeding drive becomes active whenever the level of the physiological variables drops below a satisfaction threshold. The robot then uses the path integration vector to return to the resource. Similarly, obstacle detection triggers the defensive drive and generates an avoidance behavior. In some case, these two low-level motivations can be contradictory, e.g. if there is an obstacle (object or other robot) on the way. A competition between the appetitive and aversive drives allows them to inhibit each other, which favours the approach or the avoidance behavior depending on the drives levels. Similarly to the affective state, we define the approach $m_{a p}$ and avoidance $m_{a v}$ motivation levels at time $t$ as following:

$$
\begin{aligned}
& m_{a p}(t)=\varepsilon_{m} \cdot m_{a p}(t-d t)-\gamma_{m} \cdot m_{a v}(t-d t) \\
& m_{a v}(t)=\varepsilon_{m} \cdot m_{a v}(t-d t)-\gamma_{m} \cdot m_{a p}(t-d t)
\end{aligned}
$$

where $\varepsilon_{m}$ and $\gamma_{m}$ respectively represent the integration and inhibition factors of the competition.

In the third experiment (Sect. VII), we evaluate our model in a different framework. Like in some psychological experiments, we consider a task in which an agent has to reach an object [14][15]. We use visual inputs to represent PPS. Therefore, a set of objects are learned by the system in a preexperiment phase. We use an algorithm for points of interest (PoI) extraction by convolving the gradient images with a DoG filter. These PoI are categorized as visual signatures and associated with the objects to learn (See [20] for more details). Our system also learns a set of landmarks that should not be associated with none of the objects in order to be robust to background "noise". During the experiment, an approach behavior is simulated by a motivation to reach recognized objects. In addition, desirable and threatening objects respectively trigger an additional appetitive motivation and a defensive avoidance behavior.

In all experiments, the architecture is run on the Promethe neural network simulator [21]. Each operation of the information processing flow (Fig. 3) can be computed as soon as the information from previous modules is updated. Independent modules are executed in parallel (separate threads). 


\section{B. Experimental paradigm}

In order to study the impact of the emotional modulation of the peripersonal space, we compare the Model version behavior with two altered versions of the architecture:

- NoCompet version: We still modulate robot PPS according to its emotional state. However, there is no lateral inhibition between punishment and reward signals nor between appetitive and aversive drives.

- NoModul version: No modulation of approach/avoidance is performed at all. Robot drives only serve for triggering homing behavior for example. This version is the closest to a classical reactive architecture. Except here approach and avoidance have the same weight in the DNF.

\section{EXPERIMENT 1: REAL ROBOT VS. STATIC OBSTACLE}

\section{A. Experimental setup}

Let us consider the $40 \mathrm{~cm}$-wide, $50 \mathrm{~cm}$-long, $140 \mathrm{~cm}$ high, 4-wheel mobile robot. It is embedded with a light sensor. The latter is placed under the robot to detect a 45 $\mathrm{cm} \times 45 \mathrm{~cm}$ red-colored zone considered as a resource. The platform also has 9 ultra-sound proximity sensors, of which we only use a subset to cover a 180 degrees-wide front field. The experiments are run in one of the rooms of our lab, approximately $8 \mathrm{~m}$ long by $6.5 \mathrm{~m}$ wide.

In this experiment: $\varepsilon_{m}=0.8, \gamma_{m}=0.2, \varepsilon_{a}=0.2$, $\gamma_{m}=0.8, \alpha_{r}=0.1$ and $\beta_{r} 0.01$.

\section{B. Results}

When the level of its physiological variable drops below the satisfaction threshold ( 0.5 in this experiment), the robot uses the return vector represented in the path integration field to go back to the resource. Fig. 4 shows multiple trajectories observed in a regular homing case as well as when the three architecture versions - Model, NoCompet and NoModul face a static obstacle (a cardboard box) put on the resource. In the Model case, the robot is able to reach the resource, even after a deviation due to its avoidance behavior. This is allowed by the inhibition of the defensive sub-behavior by the appetitive one when the level of the physiological variable becomes critical. On the other hand, with the two other architecture, the robot fails to feed. Either the defensive drive dominates the robot behavior (NoCompet version, and green and blue trajectories with NoModul version) or the latter oscillates between approach and avoidance (purple trajectories with NoModul version).

\section{Discussion}

With the NoCompet version, there is an implicite prioritization of the sub-behaviors due to their respective dynamics. Thus, the defensive drive has a bigger impact on robot behavior. The appetitive drive increases more slowly. When, it's high enough it can only cancel the avoidance behavior, making the robot stop in front of the obstacle.

In the NoModul case, the robot either stops in front of the obstacle or diverges. Depending on the situation, if the approach and the avoidance fields are perfectly opposed,

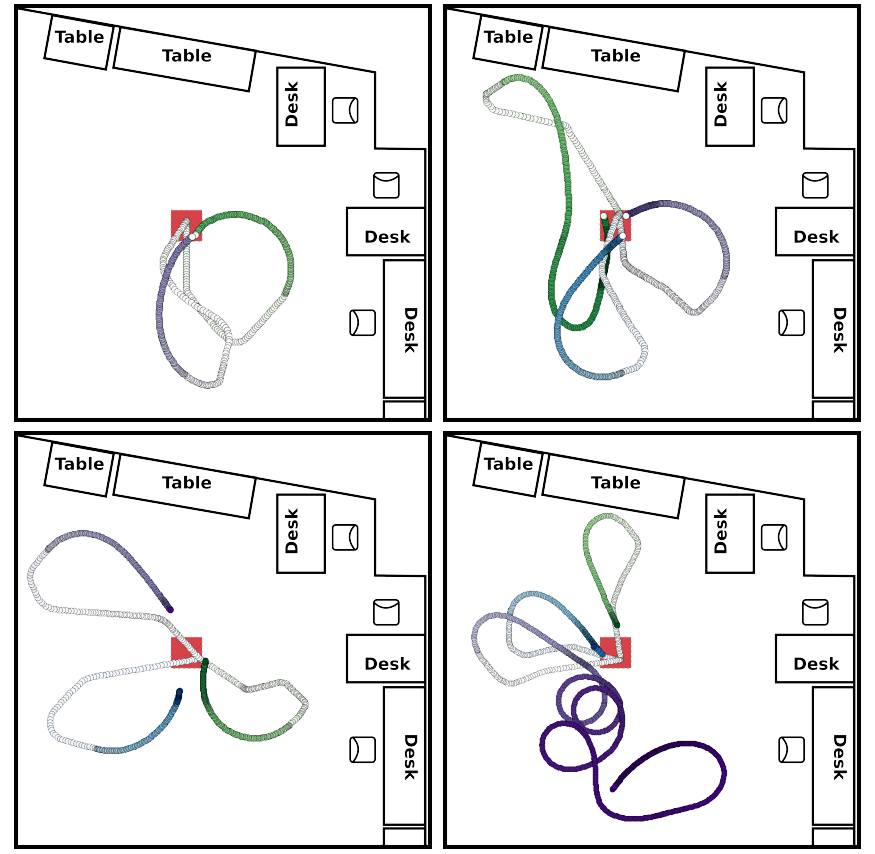

Fig. 4. Experiment 1 results. TOP-LEFT: Robot trajectories in a regular case of homing toward the resource. Colored gradients from light to dark represent the decay of the phyiological variable. White dots at the end of the trajectories indicate a new ingestion. TOP-RIGHT: Trajectories where the robot is able to reach the resource by pushing the obstacle (Model version). BOTTOM-LEFT: Trajectories where the robot stops before reaching the resource (obstacle-induced deadlock with NoCompet version). BOTTOMRIGHT: Trajectories where the robot stops or diverges before reaching the resource (obstacle-induced deadlock with NoModul version).

they cancel each other quite quickly. Otherwise, the system oscillates between the attractor and the distractor respectively created by the appetitive and the aversive sub-behavior. Thus, the robot accumulates errors in the path integration fields and is unable to reach the resource.

On the other hand, with the Model version, the approach sub-behavior can inhibit the defensive one when the level of the physiological variable becomes too low. Even if the robot deviates from the resource due to the avoidance mechanism, it is able to access the resource afterwards.

Moreover, one should note that in the NoCompet case, deadlocks occur farther from the resource in comparison with the NoModul architecture. This is due to the robot extending its comfort zone because of negative affective states induced by collision with the box.

\section{EXPERIMENT 2: MULTIROBOT COMPETITION SIMULATION}

\section{A. Experimental setup}

This experiment is performed on the Webots simulator in order to avoid damaging real robots. We simulate two robots identical to the one described previously moving in a $17.5 \mathrm{~m} \times 15 \mathrm{~m}$ environment. In this experiment, $\alpha_{r}=0.05$, meaning the resource ingestion is slower than in the previous experiment in order to observe the competition between the two robots. Others parameters are kept as in experiment 1. 

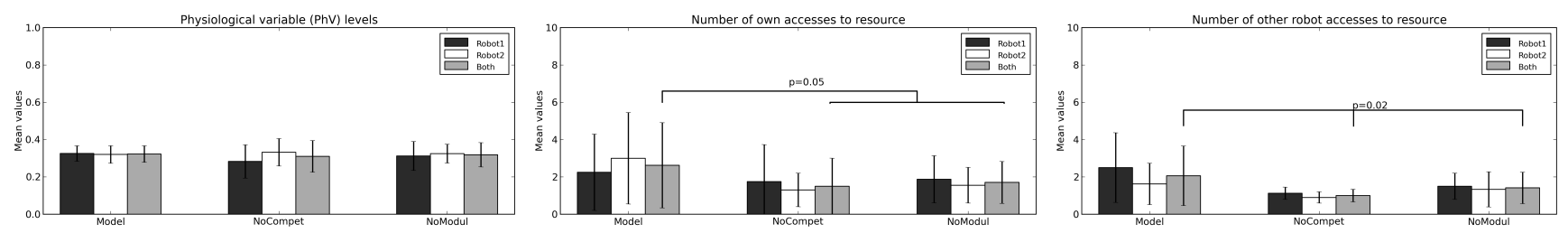

Fig. 5. Experiment 2 results: Statistical significance of the effect of different architecture versions on the considered measures. No significant difference in terms of food depletion is found but the alternation in the access to the resource varies among the architecture versions. A strong tendency is found with model variable on nb_own_access as well as a significant effect of version on nb_other_access

We define a cycle as an interval in which a robot, initially satisfied (non-hungry), consumes the energy obtained from the previous ingestion and returns to the resource in order to feed once again. Each of these cycles is considered as an independant sample of the multirobot competition for the resource. Once its feeding drive satisfied, a robot get away from the resource. It randomly navigates in the environment updating its path integration field to be able to return to the resource when needed. Similarly to the previous experiment, with NoModul and NoCompet versions, robots are expected to be unable to access the resource until the other is done feeding. However, in the Model version, robots should be able to fight for the resource by pushing each other.

To test our hypothesis, we use three measures:

- min_phyvar: Lowest level of the physiological variable associated with the feeding drive,

- nb_own_access: Number of own accesses to the resource within a full cycle,

- nb_other_access: Number of other robot accesses to the resource within own cycle.

The first one is a measure of food deplation, i.e. how close to starvation the robots get. The two latter quantify cycle interruptions. Besides, we consider two variables:

- model: Whether our model is used (the NoCompet and NoModul versions are gathered in one group),

- version: Which version is used (each of the 3 versions is association with a different group).

\section{B. Results}

We compare the 3 architecture versions in 15-minute simulations ( $N=51, N_{\text {Model }}=16, N_{\text {NoCompet }}=18, N_{\text {NoModul }}=17$; min_phyvar: $\mu=0.68, \sigma=0.07$; nb_own_access: $\mu=1.92$, $\sigma=1.78$ nb_other_access: $\mu=1.47, \sigma=1.14)$. None of the variable follows a normal distribution.

Kruskal-Wallis non-parametric test shows that there is no effect of version nor model on min phyvar (resp. Chi ${ }^{2}=$ $0.45, p=0.80$; and $C h i^{2}=0.00, p=0.95$ ). No significant effect of version on $n b \_$own_access was found either $\left(C h i^{2}=\right.$ $5.36, p=0.07)$. However, there is a strong tendency with model $\left(C h i^{2}=3.81, p=0.05\right)$. Also, there is a main effect of both model and version on nb_other_access (resp. Chi ${ }^{2}=$ 6.03, $p=0.01$; and $C h i^{2}=8.19, p=0.02$ ).

\section{Discussion}

The results regarding the min_phyvar measure show there is no significant difference between the architecture version in terms of food deplation. This is due to the random exploration following feeding phases and to resource consumption being slower that its ingestion. This leaves the possibility for the robot to alternate in resource access. Yet, it is interesting to observe how this alternation occurs, i.e. how the robot interact in this survival task. Indeed, there is a social dimension that emerges from the emotional modulation of the robot peripersonal space.

nb_own_access and nb_other_access allow to caracterize the alternation in resource access. In addition, let us consider two additional measures bin(nb_own_access) and bin(nb_other_access) respectively equal to 1 if $n b \_o w n \_a c c e s s$ and $n b \_o t h e r \_a c c e s s$ are greater than 1 , and 0 otherwise. Indeed, in the case of a perfect alternation of the robots over the resource, each should access it exclusively and only once in every cycle. Any different configuration could correspond to a feeding cycle being interrupted by another robot. In this case, we find a strong tendency on bin(nb_own_access) with both model and version (resp. $C h i^{2}=3.68, p=0.05$; and $\left.C h i^{2}=6.01, p=0.05\right)$ as well as a significant effect on bin(nb_other_access) (resp. $C h i^{2}=5.19, p=0.02$; and $\left.C h i^{2}=6.73, p=0.03\right)$.

Similarly to Experiment 1 (Sect. V), the robots tend to be unable to access the resource before it is free when the NoCompet or NoModul architecture is used. In the NoModul case, collisions between the robots occur due the appetitive and aversive behaviors having exactly the same weight. Therefore, some cycle interruptions can happen. Yet, the robot generally deviates from the resource in order to avoid the other robot currently feeding. We argue that interactions between the robots can be seen as emotionally and socially communicative in the NoCompet and Model versions. With the former, robots seem either patient or fearful. Their modulation of their PPS make them extend their comfort zone. They are more sensitive to aversive stimuli and defensive sub-behavior tend to take over the appetitive one. On the contrary, using the Model version, the robots seems more proactive and determined. When the resource is not available they try to push whatever is on their way. In both architectures, the robots build a representation of their reachable and comfort areas. They also interact in a way that expresses aspects of their internal states. However, when the appetitive and aversive motivations cannot inhibit each one another, it can lead to deadlock situations like in Experiment 1 (Sect. V). 


\section{EXPERIMENT 3: TOWARD VISION-BASED OBJECT REACHING}

\section{A. Experimental setup}

In this experiment, we use a 60 degrees firewire camera. The latter is directed toward a platform where the experimenter puts objects that our system is asked to reach (see Fig. 6). Among the latter, one is desirable (toy), one is neutral (cable roll) and one is threatening (snap-off utility knife). The head direction field is used to integrate visual stimuli i.e. recognized landmarks - in the perception of the robot peripersonal space. We simulate an approach motivation (reaching) and compare how their reachability is perceived by the robot depending on their desirability. That is to say, the level of distortion induced on the robot reachable space induced by the detection of these objects.

\section{B. Results}

Fig. 7 shows how reachability perception varies from one object to another using the three architectures. In our system, the reachability depends on the object recognition level. Therefore, in the following, we will consider reachability perception proportionally to a baseline. The latter corresponds to an estimation of reachability only determined by the object recognition level.

Using the Model version, the desirable object seem more reachable than the neutral one. Also, the threatening object is perceived as less reachable due to the harm aversion inhibiting the approach behavior. On the contrary, in the NoCompet case, the negative one reachability in not altered by its aversive caracteristics since no competition between motivations occurs. However, similarly to Model, the positively valenced object is indeed perceived as more reachable than the others. Finally, the NoModul version perceives reachability in a rather binary way because no modulation of PPS is operated.

\section{Discussion}

In a framework that is similar to some psychological studies [14][15], our model succeeds in accounting for the objects desirability in their reachability perception. Thanks to the emotional modulation and the competition between aversion and approach, positive and negative objects respectively seem more and less reachable than neutral ones. In addition, this experiment shows how visual sensory input can be used in our architecture. In future work, objects to reach or to avoid can be recognized visually. For example, in navigation tasks like in experiments 1 and 2, such additional sensory stimuli can be merged with path integration and proximity detection in order to build robot PPS.

However, we note that if several learned objects were presented simultaneously, the system response would depend on their spatial position. If far from each other, their reachability could be perceived separately and according to their desirability. But if too close, the DNF could fuse all stimuli and could lead to an erroneous perception. This is one of the DNF limitations.
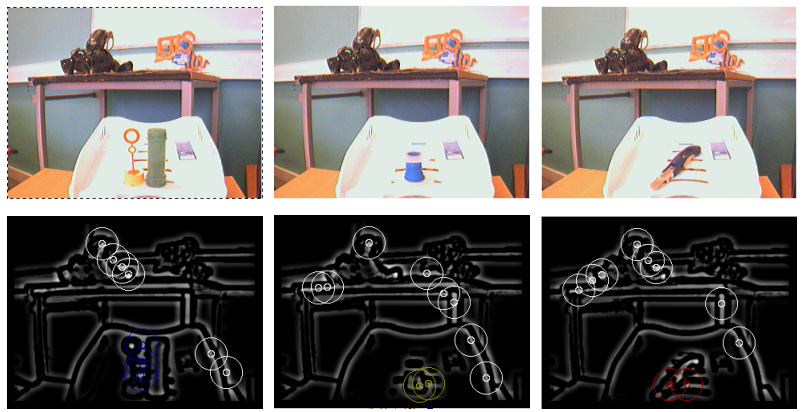

Fig. 6. Experiment 3 setup and visual object recognition. TOP: 3 objects are put in front of the camera: a toy (positive/desirable), a cable roll (neutral) and a snap-off utility knife (negative/threatening). BOTTOM: Gradient image convoluted with DoG used to detect landmark. Localviews of the desirable, neutral and threatening objects are recognized. They are represented by blue, yellow and red circles respectively. Several other points of interest are detected on background objects but are not relevant to the task (white circles).
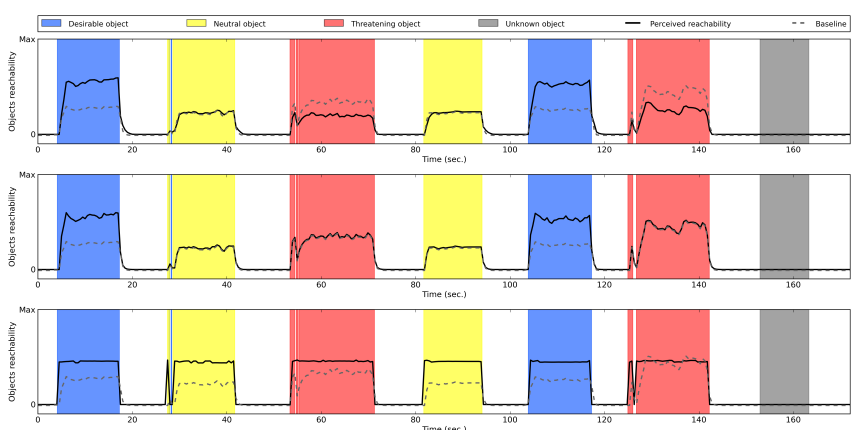

Fig. 7. Experiment 3 results: Perception of the recognized objects reachability with the three architectures. Our architectures perceives reachability depending on the object recognition level. Therefore, results should be seen in comparison with the baseline. The latter corresponds to an estimation of reachability only determined by the object recognition level. TOP: Model version. Positive and negative objects respectively seem more and less reachable than neutral ones. CENTER: NoCompet version. The desirable object seems more reachable than the other. However, neutral and threatening one reachability are perceived equally. BOTTOM: NoModul version. Reachability is quite binary, only depending on whether an object is recognized. In all cases, an unknown object is not considered in the reaching task and therefore do not alters the robot peripersonal space.

\section{GENERAL DISCUSSION}

In this paper, we propose a model in which emotions modulate the robot perception of the surrounding space. In the literature, the notion of comfort zone refer to the space needed to feel comfortable while the term reachable space is used in action-related contexts. We see these two notions as two aspects of the same process of integrating information to represent the space around oneself: the peripersonal space. Evidence of the effect of emotions on such perception can be found in psychological and neuroscientific studies. In our case, the robot emotional states depend on the dynamics of basic components: pleasure, pain and motivation. They modulate the perception of sensory input before and after they are integrated in a working memory. Thereby, we obtain a subjective and motivated representation of the surrounding space which is used to drive robots behavior.

We test this approach in two different tasks, comparing the 
results of our model with two altered versions. Experiments 1 and 2 address the single-resource problem. We highlight the role of the emotional modulation and the competition between the approach and avoidance behaviors in allowing to use PPS representation to control the robot. In experiment 3 , we show how our model accounts for desirability impact on reachability perception as it has been studied in some psychological experiments [14][15]. All together, these experiments demonstrate the generic aspect of our model, in terms of stimuli types and tasks nature.

Nevertheless, in some cases, the competition mechanism we use between appetitive and aversive sub-behaviors could be insufficient. For example, avoidance inhibition by the appetitive drive would be irrelevant in case there is a wall between the robot and the resource. Thus, our architecture needs to implement learning mechanisms that could take punishments and rewards into account in generating robot motivational states. Another option is the use of neuromodulatory signals to bias approach and avoidance like in [22].

In our approach, we took inpiration from information coding in biological systems. Indeed, several evidence of neural population coding can be found in the literature [23][24]. We also chose to uniformly use this form of coding for sensory information and motor control, as well as all intermediate representations like the working memory and the peripersonal space. Our code could directly be exploited in the action selection process. In addition, relying on a DNF to merge representations of the reachable and comfort areas allowed us to take advantages from its properties. Indeed, DNF are good candidates for robotic control because of the stability given by the fusion and bifurcation properties.

Our model relies on previous work that proposed a solution to build and update a limited working memory. In [17], the latter only handled multiple path integration vectors. The model we present in this paper suggests that the same mechanism can be used for various sensory input in addition to displacement vectors. In this work, for the sake of simplicity, different sensory modalities are integrated in separate sub-parts of the working memory. However, we could use the working memory homogeneously and make the gating mechanisms handle fields recruitment. However, we have to make sure fields are allocated to each modality.

This work highlights the role of emotions in behavior control and argue for the design of robotic architecture that rely on such mechanisms. But emotions do have a communication role as well. We believe that displaying emotionally colored behaviors foster robots acceptability. In this paper, we described how social and emotional caracteristics emerge from the way two of our robots interact. They can be perceived as being aggressive or fearful and patient depending on the way they behave in the single-resource task. Adding learning mechanisms - e.g. reward-based conditionning would allow to emphasize these caracteristics in addition to allowing the system to inhibit irrelevant behaviors. In [7], an emotional metacontroller modulates strategies weights for action selection based on novelty detection. Such module could also trigger or neuromodulate the robot learning.

\section{REFERENCES}

[1] V. Braitenberg, Vehicles: Experiments in Synthetic Psychology, 1986.

[2] R. Brooks, "A robust layered control system for a mobile robot," IEEE Journal on Robotics and Automation, vol. 2, no. 1, 1986.

[3] T. Tyrrell, "The Use of Hierarchies for Action Selection," pp. 387-420, 1993.

[4] P. Maes and R. A. Brooks, "Learning to Coordinate Behaviors." in AAAI, 1990, pp. 796-802.

[5] L. Dollé, D. Sheynikhovich, B. Girard, R. Chavarriaga, and A. Guillot, "Path planning versus cue responding: A bio-inspired model of switching between navigation strategies," Biological Cybernetics, vol. 103, no. 4, pp. 299-317, 2010.

[6] G. Schöner, M. Dose, and C. Engels, "Dynamics of behavior: Theory and applications for autonomous robot architectures," pp. 213-245, 1995.

[7] A. Jauffret, M. Belkaid, N. Cuperlier, P. Gaussier, and P. Tarroux, "Frustration as a way toward autonomy and self-improvement in robotic navigation," in 2013 IEEE 3rd Joint International Conference on Development and Learning and Epigenetic Robotics, ICDL 2013 Electronic Conference Proceedings, 2013.

[8] Michael Inzlicht and Bruce D. Bartholow and Jacob B. Hirsh, "Emotional foundations of cognitive control," Trends in Cognitive Sciences, vol. 19, no. 3, pp. 126-132, 2015.

[9] L. Pessoa, "On the relationship between emotion and cognition," Nature Reviews Neuroscience, vol. 9, no. 2, pp. 148-158, 2008.

[10] D. Cañamero, "Modeling motivations and emotions as a basis for intelligent behavior," in Proceedings of the first international conference on Autonomous agents AGENTS 97, no. Abbott 1884. ACM Press, 1997, pp. 148-155.

[11] J. Hirth, T. Braun, and K. Berns, "Emotion Based Control Architecture for Robotics Applications," in Proceedings of the 30th annual German conference on Advances in Artificial Intelligence. Springer-Verlag, 2007, pp. 464-467.

[12] D. P. Kennedy, J. Gläscher, J. M. Tyszka, and R. Adolphs, "Personal space regulation by the human amygdala." Nature neuroscience, vol. 12, no. 10, pp. 1226-1227, 2009.

[13] A. Tajadura-Jiménez, G. Pantelidou, P. Rebacz, D. Västfjäll, and M. Tsakiris, "I-space: The effects of emotional valence and source of music on interpersonal distance," PLOS ONE, vol. 6, no. 10, 2011.

[14] B. Valdés-Conroy, F. J. Román, J. a. Hinojosa, and S. P. Shorkey, "So far so good: emotion in the peripersonal/extrapersonal space." PloS one, vol. 7, no. 11, p. e49162, 2012.

[15] Y. Coello, J. Bourgeois, and T. Iachini, "Embodied perception of reachable space: how do we manage threatening objects?" pp. 131135, 2012.

[16] A. R. Damasio, Looking for Spinoza: Joy, sorrow, and the feeling brain, 2003.

[17] C. Hasson and P. Gaussier, "Path integration working memory for multi tasks dead reckoning and visual navigation," in Proceedings of the 11th international conference on Simulation of adaptive behavior: from animals to animats. Springer-Verlag, 2010, pp. 380-389.

[18] C. Hasson, "Modeling emotional mechanisms for an autonomous robot: A developmental and social perspective," Ph.D. dissertation, 2011.

[19] E. Comoli, P. Das Neves Favaro, N. Vautrelle, M. Leriche, P. G. Overton, and P. Redgrave, "Segregated Anatomical Input to SubRegions of the Rodent Superior Colliculus Associated with Approach and Defense," 2012.

[20] R. Braud, G. Mostafaoui, A. Karaouzene, and P. Gaussier, "Simulating the emergence of early physical and social interactions: A developmental route through low level visuomotor learning," in From Animals to Animats 13. Springer, 2014, pp. 154-165.

[21] M. Lagarde, P. Andry, and P. Gaussier, "Distributed real time neural networks in interactive complex systems," in CSTST, 2008, pp. 95100.

[22] J. L. Krichmar, "A neurorobotic platform to test the influence of neuromodulatory signaling on anxious and curious behavior." Frontiers in neurorobotics, vol. 7, no. February, p. 1, 2013.

[23] J. S. Taube, R. U. Muller, and J. B. Ranck, "Head-direction cells recorded from the postsubiculum in freely moving rats. I. Description and quantitative analysis." The Journal of neuroscience, vol. 10, no. 2, pp. 420-435, 1990.

[24] E. Bullmore and O. Sporns, "Complex brain networks: graph theoretical analysis of structural and functional systems." Nature reviews. Neuroscience, vol. 10, no. 3, pp. 186-98, 2009. 